\title{
Applications of the Lefschetz Number to Digital Images
}

\author{
Ozgur Ege* Ismet Karaca
}

\begin{abstract}
The goal of this paper is to develop some applications of the Lefschetz fixed point theorem to digital images. We also deal with relative and reduced Lefschetz fixed point theorem for digital complexes. We give some examples related to the topic. We calculate the degree of the antipodal map for spherelike digital images using fixed point properties.
\end{abstract}

\section{Introduction}

Digital topology with algebraic properties is a growing area in computer vision, image processing and fixed point theory. Many researchers, such as Rosenfeld, Kong, Kopperman, Kovalevsky, Boxer, Karaca, Han and others, have studied the properties of digital images using topology and algebraic topology.

The Lefschetz fixed point theorem counts fixed points of a continuous mapping from a compact topological space $X$ to itself via traces of the induced mappings on the homology groups of $X$. The Lefschetz number has been used in order to treat fixed point theory. Since the Lefschetz number is a homotopy invariant, it can be used to classify digital images. The main advantage is that this number can be easily computed.

Arslan et al. [1] introduce the simplicial homology groups of $n$-dimensional digital images. Boxer et al. [7] expanded knowledge of the simplicial homology

\footnotetext{
${ }^{*}$ Corresponding Author.

Received by the editors in February 2014.

Communicated by Y. Félix.

2010 Mathematics Subject Classification : 55N35, 55M20, 68R10, 68U05, 68U10.

Key words and phrases : Digital image, the Lefschetz Fixed Point Theorem, the Euler Characteristic, Lefschetz number.
} 
groups of digital images. They study the simplicial homology groups of certain minimal simple closed surfaces, extended an earlier definition of the Euler characteristics of a digital image, and computed the Euler characteristic of several digital surfaces.

Karaca and Ege [14] study some results related to the simplicial homology groups of $2 D$ digital images. They show that if a bounded digital image $X \subset \mathbb{Z}$ is nonempty and $\kappa$-connected, then its homology groups at the first dimension are trivial. They also prove that the homology groups of the operands of a wedge of digital images are not necessarily additive. Ege and Karaca [11] give characteristic properties of the simplicial homology groups of digital images and investigate the Eilenberg-Steenrod axioms for the simplicial homology groups of digital images. Ege and Karaca [10] construct Lefschetz fixed point theory for digital images and get some nice results. Ege et al. [12] study relative homology groups of digital images and compute relative homology groups of some digital images.

This paper is organized as follows. The second section provides the general notions of digital images with $\kappa$-adjacency relations, digital homotopy and homology groups. In Section 3, we provide some important applications about the Lefschetz number, relative and reduced versions of the Lefschetz number, and we present some examples. In the final Section we draw some conclusions.

\section{Preliminaries}

Let $\mathbb{Z}$ be the set of integers. A digital image is a pair $(X, \kappa)$, where $X \subset \mathbb{Z}^{n}$ for some positive integer $n$ and $\kappa$ represents a certain adjacency relation for the elements of $X$. There are various adjacency relations in the study of digital images but we give only one of them. Let $l, n$ be positive integers, $1 \leq l \leq n$ and two distinct points $p=\left(p_{1}, p_{2}, \ldots, p_{n}\right), q=\left(q_{1}, q_{2}, \ldots, q_{n}\right)$ in $\mathbb{Z}^{n}, p$ and $q$ are $k_{l}$-adjacent [5], if there are at most $l$ distinct coordinates $j$ for which $\left|p_{j}-q_{j}\right|=1$ and for all other coordinates $j, p_{j}=q_{j}$. The number of points $q \in \mathbb{Z}^{n}$ that are adjacent to a given point $p \in \mathbb{Z}^{n}$ is represented by a $k_{l}$-adjacency relation. From this viewpoint, the $k_{1}$-adjacency on $\mathbb{Z}$ is denoted by the number 2 and $k_{1}$-adjacent points are called 2-adjacent. In a similar way, we call 4-adjacent and 8-adjacent for $k_{1}$ and $k_{2}$-adjacent points of $\mathbb{Z}^{2}$; and in $\mathbb{Z}^{3}, 6$-adjacent, 18-adjacent and 26-adjacent for $k_{1}, k_{2}$ and $k_{3}$-adjacent points, respectively.

A $\kappa$-neighbor of $p \in \mathbb{Z}^{n}$ is a point of $\mathbb{Z}^{n}$ which is $\kappa$-adjacent to $p$, where $\kappa$ is an adjacency relation defined on $\mathbb{Z}^{n}$. A digital image $X \subset \mathbb{Z}^{n}$ is $\kappa$-connected [13] if and only if for every pair of different points $x, y \in X$, there is a set $\left\{x_{0}, x_{1}, \ldots, x_{r}\right\}$ of points of a digital image $X$ such that $x=x_{0}, y=x_{r}$ and $x_{i}$ and $x_{i+1}$ are $\kappa$-neighbors where $i=0,1, \ldots, r-1$. A set of the form

$$
[a, b]_{\mathbb{Z}}=\{z \in \mathbb{Z} \mid a \leq z \leq b\},
$$

is said to be $a$ digital interval [2], where $a, b \in \mathbb{Z}$ with $a<b$.

Let $\left(X, \kappa_{0}\right) \subset \mathbb{Z}^{n_{0}}$ and $\left(Y, \kappa_{1}\right) \subset \mathbb{Z}^{n_{1}}$ be digital images. A function $f: X \longrightarrow Y$ is called $\left(\kappa_{0}, \kappa_{1}\right)$-continuous [3] if for every $\kappa_{0}$-connected subset $U$ of $X, f(U)$ is a $\kappa_{1}$-connected subset of $Y$. 
In a digital image $(X, \kappa)$, if there is a $(2, \kappa)$-continuous function $f:[0, m]_{\mathbb{Z}} \longrightarrow$ $X$ such that $f(0)=x$ and $f(m)=y$, then we say that there exists $a$ digital $\kappa$-path [6] from $x$ to $y$. If $f(0)=f(m)$ then $f$ is called digital $\kappa$-loop and the point $f(0)$ is the base point of the loop $f$. When a digital loop $f$ is a constant function, it is said to be a trivial loop. A simple closed $\kappa$-curve of $m \geq 4$ points in a digital image $X$ is a sequence $\{f(0), f(1), \ldots, f(m-1)\}$ of images of the $\kappa$-path $f:[0, m-1]_{\mathbb{Z}} \longrightarrow X$ such that $f(i)$ and $f(j)$ are $\kappa$-adjacent if and only if $j=i \pm \bmod m$.

Definition 2.1. Let $\left(X, \kappa_{0}\right) \subset \mathbb{Z}^{n_{0}}$ and $\left(Y, \kappa_{1}\right) \subset \mathbb{Z}^{n_{1}}$ be digital images. A function $f: X \longrightarrow Y$ is $a\left(\kappa_{0}, \kappa_{1}\right)$-isomorphism [1] if $f$ is $\left(\kappa_{0}, \kappa_{1}\right)$-continuous and bijective and $f^{-1}: Y \longrightarrow X$ is $\left(\kappa_{1}, \kappa_{0}\right)$-continuous and it is denoted by $X \approx_{\left(\kappa_{0}, \kappa_{1}\right)} Y$.

For a digital image $(X, \kappa)$ and its subset $(A, \kappa)$, we call $(X, A)$ a digital image pair with $\kappa$-adjacency. If $A$ is a singleton set $\left\{x_{0}\right\}$, then $\left(X, x_{0}\right)$ is called a pointed digital image.

Definition 2.2. [3]. Let $\left(X, \kappa_{0}\right) \subset \mathbb{Z}^{n_{0}}$ and $\left(Y, \kappa_{1}\right) \subset \mathbb{Z}^{n_{1}}$ be digital images. Two $\left(\kappa_{0}, \kappa_{1}\right)$-continuous functions $f, g: X \longrightarrow Y$ are said to be digitally $\left(\kappa_{0}, \kappa_{1}\right)$ homotopic in $Y$ if there is a positive integer $m$ and a function $H: X \times[0, m]_{\mathbb{Z}} \longrightarrow$ $Y$ such that

- for all $x \in X, H(x, 0)=f(x)$ and $H(x, m)=g(x)$;

- for all $x \in X$, the induced function $H_{x}:[0, m]_{\mathbb{Z}} \longrightarrow Y$ defined by

$$
H_{x}(t)=H(x, t) \quad \text { for all } t \in[0, m]_{\mathbb{Z}},
$$

is $\left(2, \kappa_{1}\right)$-continuous; and

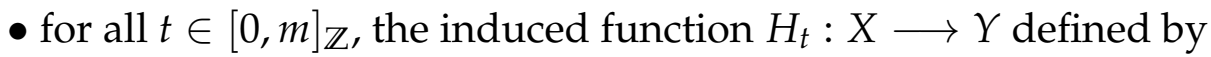

$$
H_{t}(x)=H(x, t) \quad \text { for all } x \in X,
$$

is $\left(\kappa_{0}, \kappa_{1}\right)$-continuous.

The function $H$ is called a digital $\left(\kappa_{0}, \kappa_{1}\right)$-homotopy between $f$ and $g$. If these functions are digitally $\left(\kappa_{0}, \kappa_{1}\right)$-homotopic, this is denoted $f \simeq_{\left(\kappa_{0}, \kappa_{1}\right)} g$. The digital $\left(\kappa_{0}, \kappa_{1}\right)$-homotopy relation [3] is equivalence among digitally continuous functions $f:\left(X, \kappa_{0}\right) \longrightarrow\left(Y, \kappa_{1}\right)$.

Definition 2.3. [3]. Let $f: X \longrightarrow Y$ and $g: Y \longrightarrow X$ be $\left(\kappa_{0}, \kappa_{1}\right)$ and $\left(\kappa_{1}, \kappa_{0}\right)$ continuous functions respectively such that

$$
f \circ g \simeq_{\left(\kappa_{1}, \kappa_{1}\right)} 1_{Y} \text { and } g \circ f \simeq_{\left(\kappa_{0}, \kappa_{0}\right)} 1_{X}
$$

We say that $X$ and $Y$ have the same $\left(\kappa_{0}, \kappa_{1}\right)$-homotopy type and that $X$ and $Y$ are $\left(\kappa_{0}, \kappa_{1}\right)$-homotopy equivalent. 
Definition 2.4. [3]. (i) Let $(X, \kappa)$ and $\left(Y, \kappa^{\prime}\right)$ be digital images. A digitally continuous function $f: X \longrightarrow Y$ is digitally nullhomotopic if $f$ is digitally homotopic in $Y$ to a constant function. A digital image $(X, \kappa)$ is $\kappa$-contractible if its identity map is digitally nullhomotopic.

(ii) Let $(X, A)$ be a digital image pair with $\kappa$-adjacency. $A$ is called a $\kappa$-retract of $X$ if and only if there is a $\kappa$-continuous function $r: X \longrightarrow A$ such that $r(a)=a$ for all $a \in A$. Then the function $r$ is called a $\kappa$-retraction of $X$ onto $A$.

Definition 2.5. [15]. Let $S$ be a set of nonempty subsets of a digital image $(X, \kappa)$. Then the members of $S$ are called simplexes of $(X, \kappa)$ if the following hold:

a) If $p$ and $q$ are distinct points of $s \in S$, then $p$ and $q$ are $\kappa$-adjacent.

b) If $s \in S$ and $\varnothing \neq t \subset s$, then $t \in S$.

An $m$-simplex is a simplex $S$ such that $|S|=m+1$.

Let $P$ be a digital $m$-simplex. If $P^{\prime}$ is a nonempty proper subset of $P$, then $P^{\prime}$ is called a face of $P$. We write $\operatorname{Vert}(P)$ to denote the vertex set of $P$, namely, the set of all digital 0 -simplexes in $P$. A digital subcomplex $A$ of a digital simplicial complex $X$ with $\kappa$-adjacency is a digital simplicial complex [15] contained in $X$ with $\operatorname{Vert}(A) \subset \operatorname{Vert}(X)$.

Definition 2.6. Let $(X, \kappa)$ be a finite collection of digital $m$-simplexes, $0 \leq m \leq d$ for some nonnegative integer $d$. If the following statements hold, then $(X, \kappa)$ is called [1] a finite digital simplicial complex :

(1) If $P$ belongs to $X$, then every face of $P$ also belongs to $X$.

(2) If $P, Q \in X$, then $P \cap Q$ is either empty or a common face of $P$ and $Q$.

The dimension of a digital simplicial complex $X$ is the largest integer $m$ such that $X$ has an $m$-simplex. $C_{q}^{\kappa}(X)$ is a free abelian group [1] with basis of all digital $(\kappa, q)$-simplexes in $X$.

Corollary 2.7. [1]. Let $(X, \kappa) \subset \mathbb{Z}^{n}$ be a digital simplicial complex of dimension $m$. Then, for all $q>m, C_{q}^{\kappa}(X)$ is a trivial group.

Let $(X, \kappa) \subset \mathbb{Z}^{n}$ be a digital simplicial complex of dimension $m$. The homomorphism $\partial_{q}: C_{q}^{\mathcal{k}}(X) \longrightarrow C_{q-1}^{\mathcal{N}}(X)$ defined (see [1]) by

$$
\partial_{q}\left(<p_{0}, p_{1}, \ldots, p_{q}>\right)= \begin{cases}\sum_{i=0}^{q}(-1)^{i}<p_{0}, p_{1}, \ldots, \hat{p}_{i}, \ldots, p_{q}>, & q \leq m \\ 0, & q>m\end{cases}
$$

is called a boundary homomorphism, where $\hat{p}_{i}$ means delete the point $p_{i}$. In [1], it is shown that for all $1 \leq q \leq m$,

$$
\partial_{q-1} \circ \partial_{q}=0
$$


Definition 2.8. [7]. Let $(X, \kappa)$ be a digital simplicial complex.

(1) $Z_{q}^{\kappa}(X)=\operatorname{Ker} \partial_{q}$ is called the group of digital simplicial $q$-cycles.

(2) $B_{q}^{\kappa}(X)=\operatorname{Im} \partial_{q+1}$ is called the group of digital simplicial $q$-boundaries.

(3) $H_{q}^{\kappa}(X)=Z_{q}^{\kappa}(X) / B_{q}^{\kappa}(X)$ is called the $q$ th digital simplicial homology group.

We recall some important examples about digital homology groups of certain digital images.

Example 2.9. Let $X=\{(0,0),(1,0),(0,1),(1,1)\} \subset \mathbb{Z}^{2}$ be a digital image with 4 -adjacency (see Figure 1). In [1], it is shown that digital homology groups of $X$ are:

$$
H_{q}^{4}(X)= \begin{cases}\mathbb{Z}, & q=0 \\ 0, & q \neq 0\end{cases}
$$

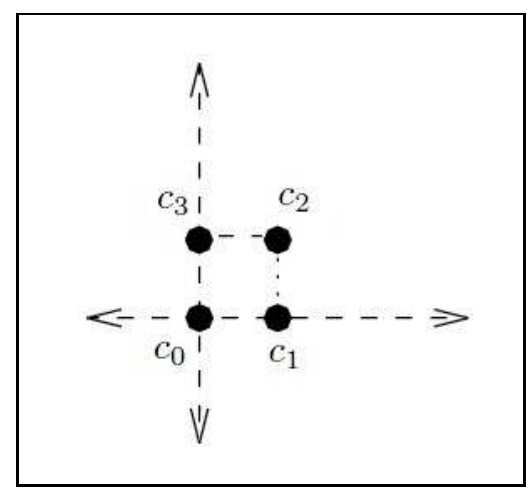

Figure 1: $X$

Example 2.10. Let $M S S_{6}^{\prime}=\left\{c_{0}=(0,0,0), c_{1}=(1,0,0), c_{2}=(1,1,0)\right.$, $\left.c_{3}=(0,1,0), c_{4}=(0,0,1), c_{5}=(1,0,1), c_{6}=(1,1,1), c_{7}=(0,1,1)\right\}$ be a digital image with 6-adjacency (see Figure 2). Boxer et al. [7] show that

$$
H_{q}^{6}\left(M_{S S_{6}^{\prime}}^{\prime}\right)= \begin{cases}\mathbb{Z}, & q=0 \\ \mathbb{Z}^{5}, & q=1 \\ 0, & q \neq 0,1\end{cases}
$$

Example 2.11. Let $M S S_{18}^{\prime}=\left\{c_{0}=(1,1,0), c_{1}=(0,2,0), c_{2}=(-1,1,0)\right.$, $\left.c_{3}=(0,0,0), c_{4}=(0,1,-1), c_{5}=(0,1,1)\right\}$ be a digital image with 18-adjacency (see Figure 3). Boxer et al. [7] get the following

$$
H_{q}^{18}\left(M S S_{18}^{\prime}\right)= \begin{cases}\mathbb{Z}, & q=0,2 \\ 0, & q \neq 0,2 .\end{cases}
$$




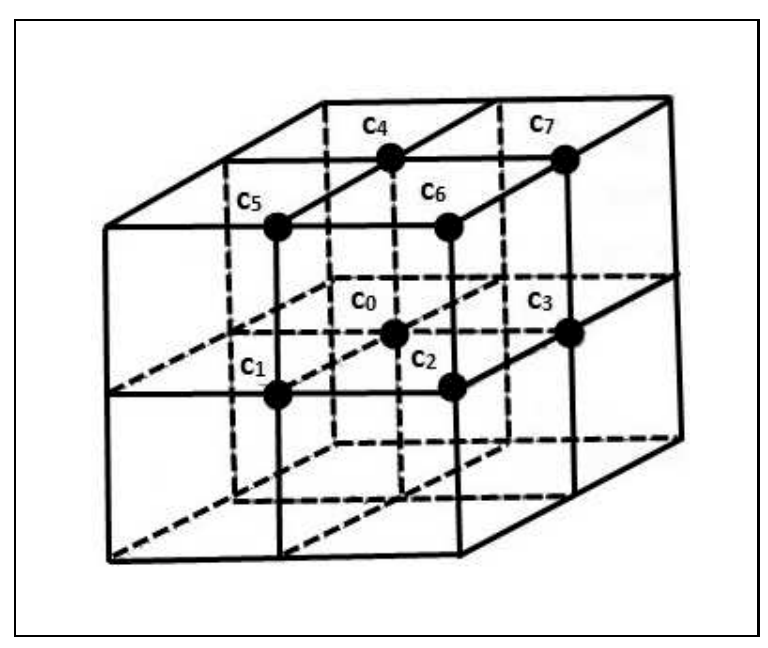

Figure 2: $\mathrm{MSS}_{6}^{\prime}$

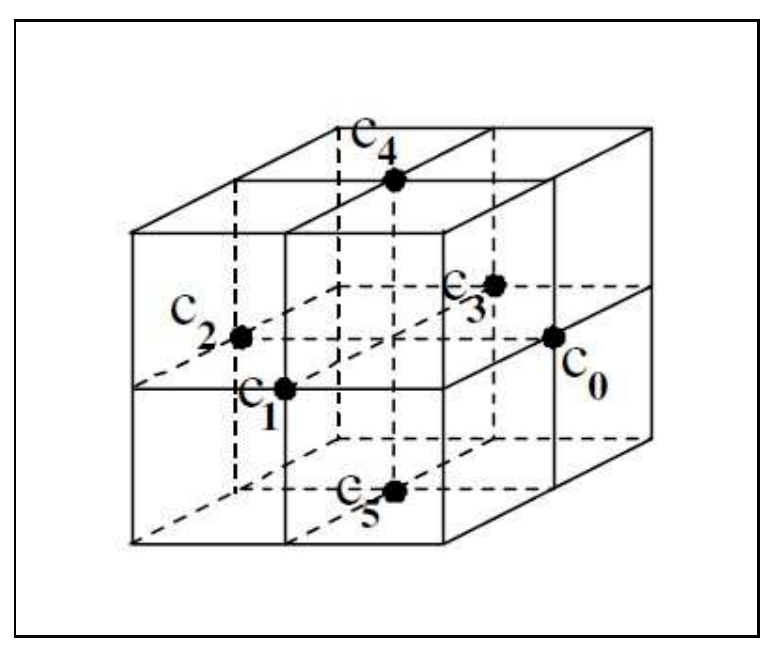

Figure 3: $M S S_{18}^{\prime}$

Theorem 2.12. [7]. Let $(X, \kappa)$ be a directed digital simplicial complex of dimension $m$.

(1) $H_{q}^{\kappa}(X)$ is a finitely generated abelian group for every $q \geq 0$.

(2) $H_{q}^{\kappa}(X)$ is a trivial group for all $q>m$.

(3) $H_{m}^{\kappa}(X)$ is a free abelian group, possible zero.

Boxer et al. [7] conclude that for each $q \geq 0, H_{q}^{\kappa}$ is a covariant functor from the category of digital simplicial complexes and simplicial maps to the category of abelian groups.

Definition 2.13. [7]. Let $f:\left(X, \kappa_{0}\right) \longrightarrow\left(Y, \kappa_{1}\right)$ be a function between two digital images. If for every digital $\left(\kappa_{0}, m\right)$-simplex $P$ determined by $\kappa_{0}$ in $X, f(P)$ is a $\left(\kappa_{1}, n\right)$-simplex in $Y$ for some $n \leq m$, then $f$ is called a digital simplicial map. 
For a digital simplicial map $f:\left(X, \kappa_{0}\right) \longrightarrow\left(Y, \kappa_{1}\right)$ and $q \geq 0$, two induced homomorphisms $f_{\#}: C_{q}^{\kappa_{0}}(X) \longrightarrow C_{q}^{\kappa_{1}}(Y)$ and $f_{*}: H_{q}^{\kappa_{0}}(X) \longrightarrow H_{q}^{\kappa_{1}}(Y)$ are defined by (see [7])

$$
\begin{gathered}
f_{\#}\left(<p_{0}, \ldots, p_{q}>\right)=<f_{\#}\left(p_{0}\right), \ldots, f_{\#}\left(p_{q}\right)>, \\
f_{*}\left(z+B_{q}^{\kappa}(X)\right)=f_{\#}(z)+B_{q}^{\kappa}(Y),
\end{gathered}
$$

where $z \in Z_{q}^{\kappa}(X)$, respectively.

Definition 2.14. [12]. Let $(A, \kappa)$ be a digital subcomplex of the digital simplicial complex $(X, \kappa)$. Then the chain group $C_{q}^{\kappa}(A)$ is a subgroup of the chain group $C_{q}^{\kappa}(X)$. The quotient group

$$
C_{q}^{\kappa}(X, A)=C_{q}^{\kappa}(X) / C_{q}^{\kappa}(A)
$$

is called the group of relative chains of $X$ modulo $A$. The boundary operator

$$
\partial_{q}: C_{q}^{\kappa}(A) \longrightarrow C_{q-1}^{\kappa}(A)
$$

is the restriction of the boundary operator on $C_{q}^{\kappa}(X)$. It induces a homomorphism

$$
C_{q}^{\kappa}(X, A) \longrightarrow C_{q-1}^{\kappa}(X, A)
$$

of the relative chain groups and this is also denoted by $\partial_{q}$.

Definition 2.15. [12]. Let $(A, \kappa)$ be a digital subcomplex of the digital simplicial complex $(X, \kappa)$.

- $Z_{q}^{\kappa}(X, A)=\operatorname{Ker} \partial_{q}$ is called the group of digital relative simplicial $q$-cycles.

- $B_{q}^{\kappa}(X, A)=\operatorname{Im} \partial_{q+1}$ is called the group of digital relative simplicial $q$-boundaries.

- $H_{q}^{\kappa}(X, A)=Z_{q}^{\kappa}(X, A) / B_{q}^{\kappa}(X, A)$ is called the $q$ th digital relative simplicial homology group.

Definition 2.16. [12]. Let $(X, \kappa)$ be a digital simplicial complex.

$$
\widetilde{H_{0}^{\kappa}}(X)=\frac{\operatorname{Ker} \epsilon}{\operatorname{Im} \partial_{1}}
$$

is called the zero dimensional reduced digital homology group of $(X, \kappa)$, where

$$
\epsilon: C_{0}^{\kappa}(X) \longrightarrow \mathbb{Z}
$$

is defined by $\epsilon(v)=1$ and $\partial_{1}: C_{1}^{\kappa}(X) \longrightarrow C_{0}^{\kappa}(X)$ is the boundary homomorphism. If $\widetilde{H_{p}^{\kappa}}(X)=H_{p}^{\kappa}(X)$ for each $p>1$, then

$$
\left\{\widetilde{H_{i}^{\kappa}}(X), i=0,1, \ldots\right\}
$$

are called the reduced digital homology groups of $(X, \kappa)$.

Theorem 2.17. [12]. For a digital simplicial complex $(X, \kappa)$, there are the following formulas which are related to reduced homology groups :

$$
\begin{gathered}
H_{0}^{\kappa}(X) \cong \widetilde{H_{0}^{\kappa}}(X) \oplus \mathbb{Z}, \\
H_{p}^{\kappa}(X)=\widetilde{H_{p}^{\kappa}}(X), \quad p \geq 1 .
\end{gathered}
$$




\section{Applications of the Lefschetz fixed point theorem to digital images}

Let $(X, \kappa)$ be a digital image and $f:(X, \kappa) \longrightarrow(X, \kappa)$ be any $(\kappa, \kappa)$-continuous function. We say the digital image $(X, \kappa)$ has the fixed point property [10] if for every $(\kappa, \kappa)$-continuous map $f: X \longrightarrow X$ there exists $x \in X$ such that $f(x)=x$. The fixed point property is preserved by any digital isomorphism, i.e., it is a topological invariant. The Lefschetz fixed point theorem determines when there exist fixed points of a map on a finite digital simplicial complex using a characteristic of the map known as the Lefschetz number. We can give a definition of trace for a digital map as in Algebraic Topology.

Definition 3.1. [9]. Given some digital map $f: X \longrightarrow X$ where $(X, \kappa)$ is a digital image, the trace of this map is defined by considering the trace of matrix representation of $f$, that is, choosing a basis for $X$ and describing $f$ as a matrix to this basis, and taking the trace of this square matrix.

Theorem 3.2. [9]. Let $(X, \kappa)$ and $\left(Y, \kappa^{\prime}\right)$ be digital images. If $f: X \longrightarrow Y$ and $g: Y \longrightarrow X$ are digital maps, then $\operatorname{tr}(g \circ f)=\operatorname{tr}(f \circ g)$.

Proof. The proof is the same as in Algebraic Topology.

Definition 3.3. [10]. For a map $f:(X, \kappa) \longrightarrow(X, \kappa)$, where $(X, \kappa)$ is a digital image whose digital homology groups are finitely generated and vanish above some dimension, the Lefschetz number $\lambda(f)$ is defined as follows:

$$
\lambda(f)=\sum_{i=0}^{n}(-1)^{i} \operatorname{tr}\left(f_{*}\right),
$$

where $f_{*}: H_{i}^{\kappa}(X) \longrightarrow H_{i}^{\kappa}(X)$ and $t r$ denotes the trace.

Theorem 3.4. [10]. If $(X, \kappa)$ is a finite digital simplicial complex, or the retract of some finite digital simplicial complex, and $f:(X, \kappa) \longrightarrow(X, \kappa)$ is a map with $\lambda(f) \neq 0$, then $f$ has a fixed point.

Theorem 3.5. Let $(X, \kappa)$ be a digital image. If $f:(X, \kappa) \longrightarrow(X, \kappa)$ has $\lambda(f) \neq 0$, then any map homotopic to $f$ has a fixed point.

Proof. Assume that $g:(X, \kappa) \longrightarrow(X, \kappa)$ is $(\kappa, \kappa)$-homotopic to $f$. Then by homotopy axiom, we have

$$
f_{*}=g_{*}: H_{*}^{\kappa}(X) \longrightarrow H_{*}^{\kappa}(X)
$$

and hence

$$
\lambda(f)=\sum_{q=0}^{n}(-1)^{q} \operatorname{tr}\left(f_{*}\right)=\sum_{q=0}^{n}(-1)^{q} \operatorname{tr}\left(g_{*}\right)=\lambda(g) .
$$

So $g$ has a fixed point. 
Boxer et al. [6] define the Euler characteristic of digital images. Let $(X, \kappa)$ be a digital image of dimension $m$, and for each $q \geq 0$, let $\alpha_{q}$ be the number of digital $(\kappa, q)$-simplexes in $X$. The Euler characteristic of $X$ is defined by

$$
\chi(X, \kappa)=\sum_{q=0}^{m}(-1)^{q} \alpha_{q} .
$$

They also prove that if $(X, \kappa)$ is a digital image of dimension $m$, then

$$
\chi(X, \kappa)=\sum_{q=0}^{m}(-1)^{q} \operatorname{rank} H_{q}^{\kappa}(X) .
$$

Proposition 3.6. [10]. Let $(X, \kappa)$ be a digital image. If a map $f:(X, \kappa) \longrightarrow(X, \kappa)$ is homotopic to the identity, then $\lambda(f)=\chi(X, \kappa)$.

Theorem 3.7. Let $(X, \kappa)$ be a digital image. If $\chi(X) \neq 0$, then any map homotopic to identity has a fixed point.

Proof. From Proposition 3.6, if $\chi(X) \neq 0$, we have $\lambda\left(1_{X}\right) \neq 0$. Let $g$ be $(\kappa, \kappa)$-homotopic to $1_{X}$. By Theorem 3.5, we have $\lambda(g) \neq 0$ and hence $g$ has a fixed point.

Theorem 3.8. Let $\left(X, \kappa_{1}\right)$ and $\left(Y, \kappa_{2}\right)$ be two digital images. If $f: X \longrightarrow Y$ and $g: Y \longrightarrow X$ are digital maps, then

$$
\lambda(g \circ f)=\lambda(f \circ g) .
$$

Proof. By Theorem 3.2, we have

$$
\begin{aligned}
\lambda(g \circ f) & =\sum_{q=0}^{n}(-1)^{q} \operatorname{tr}\left((g \circ f)_{*}\right) \\
& =\sum_{q=0}^{n}(-1)^{q} \operatorname{tr}\left(g_{*} \circ f_{*}\right) \\
& =\sum_{q=0}^{n}(-1)^{q} \operatorname{tr}\left(f_{*} \circ g_{*}\right) \\
& =\sum_{q=0}^{n}(-1)^{q} \operatorname{tr}\left((f \circ g)_{*}\right) \\
& =\lambda(f \circ g) .
\end{aligned}
$$

Theorem 3.9. Let $\left(X, \kappa_{1}\right)$ and $\left(Y, \kappa_{2}\right)$ be two digital images. If $f: X \longrightarrow X$ is a map and $h: X \longrightarrow Y$ is a digital homotopy equivalence with a homotopy inverse $k: Y \longrightarrow X$, then

$$
\lambda(f)=\lambda(h \circ f \circ k) .
$$

Proof. From the digital homotopy equivalence of $h$ and $k$, we have

$$
h \circ k \simeq_{\left(\kappa_{2}, \kappa_{2}\right)} 1_{Y} \text { and } k \circ h \simeq_{\left(\kappa_{1}, \kappa_{1}\right)} 1_{X} .
$$


Since $k \circ h \simeq_{\left(\kappa_{1}, \kappa_{1}\right)} 1_{X}$, we get

$$
k \circ h \circ f \simeq_{\left(\kappa_{1}, \kappa_{1}\right)} 1_{X} \circ f \simeq_{\left(\kappa_{1}, \kappa_{1}\right)} f .
$$

Using Theorem 3.8, we conclude that

$$
k \circ h \circ f \simeq_{\left(\kappa_{1}, \kappa_{1}\right)} h \circ f \circ k \simeq_{\left(\kappa_{1}, \kappa_{1}\right)} f .
$$

As a result, we have $\lambda(f)=\lambda(h \circ f \circ k)$.

The boundary $B d\left(I^{n+1}\right)$ of an $(n+1)$-cube $I^{n+1}$ is homeomorphic to $n$-sphere $S^{n}$. This allows us to represent a digital sphere by using the boundary of a digital cube. We use $0_{n}$ to denote the origin of $\mathbb{Z}^{n}$. Boxer [6] defines sphere-like digital image as follows:

$$
S_{n}=[-1,1]_{\mathbb{Z}}^{n+1} \backslash\left\{0_{n+1}\right\} \subset \mathbb{Z}^{n+1} \text {. }
$$

For instance,

$$
\begin{gathered}
S_{1}=\left\{c_{0}=(1,0), c_{1}=(1,1), c_{2}=(0,1), c_{3}=(-1,1), c_{4}=(-1,0), c_{5}=(-1,-1),\right. \\
\left.c_{6}=(0,-1), c_{7}=(1,-1)\right\}
\end{gathered}
$$

is digital 1-sphere with 4 -adjacency in $\mathbb{Z}^{2}$ (see Figure 4 ).

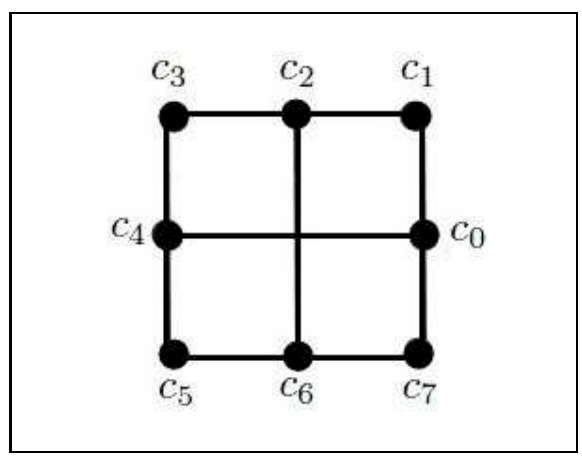

Figure 4: Digital 1-sphere $S_{1}$

The following result is given in [1].

$$
H_{q}^{4}\left(S_{1}\right)= \begin{cases}\mathbb{Z}, & q=0,1 \\ 0, & q \neq 0,1 .\end{cases}
$$

$S_{2}$, called $M S S_{6}$, is a digital image with 6-adjacency in $\mathbb{Z}^{3}$ where

$$
\begin{aligned}
S_{2}=\left\{c_{0}\right. & =(-1,-1,0), c_{1}=(0,-1,0), c_{2}=(1,-1,0), c_{3}=(1,0,0), c_{4}=(0,0,0), \\
& c_{5}=(-1,0,0), c_{6}=(-1,1,0), c_{7}=(0,1,0), c_{8}=(1,1,0), c_{9}=(1,1,1), \\
& c_{10}=(0,1,1), c_{11}=(-1,1,1), c_{12}=(-1,0,1), c_{13}=(1,0,1), c_{14}=(1,-1,1), \\
c_{15} & =(0,-1,1), c_{16}=(-1,-1,1), c_{17}=(-1,-1,2), c_{18}=(0,-1,2), \\
& c_{19}=(1,-1,2), c_{20}=(1,0,2), c_{21}=(0,0,2), c_{22}=(-1,0,2), c_{23}=(-1,1,2),
\end{aligned}
$$




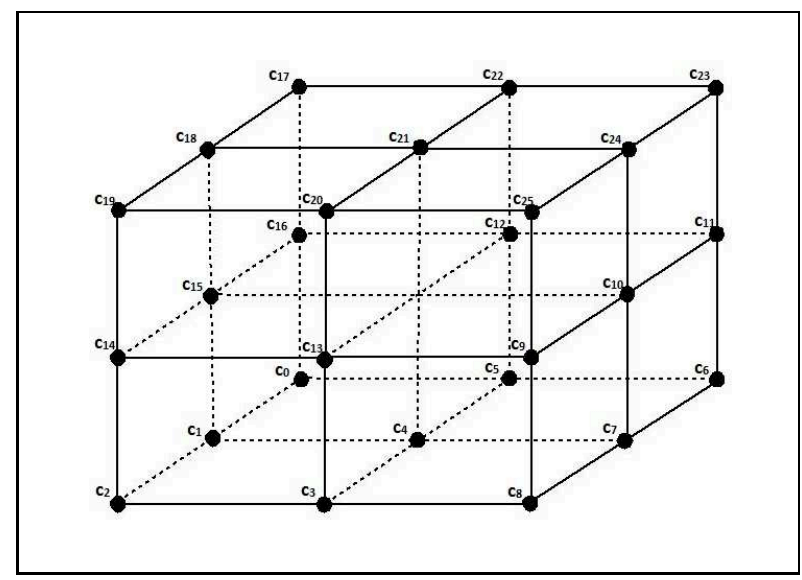

Figure 5: $S_{2}$

$$
\left.c_{24}=(0,1,2), c_{25}=(1,1,2)\right\}
$$

(see Figure 5). Demir and Karaca [8] show that the digital homology groups of $S_{2}$ are as follows:

$$
H_{q}^{6}\left(S_{2}\right)= \begin{cases}\mathbb{Z}, & q=0 \\ \mathbb{Z}^{23}, & q=1 \\ 0, & q \neq 0,1 .\end{cases}
$$

Definition 3.10. Let $f:\left(S_{n}, \kappa_{n}\right) \longrightarrow\left(S_{n}, \kappa_{n}\right)$ be a $\left(\kappa_{n}, \kappa_{n}\right)$-continuous map where $\left(S_{n}, \kappa_{n}\right)$ is digital $n$-sphere, $n \in\{1,2\}$ and $\kappa_{1}=4, \kappa_{2}=6$. Then $f$ induces homomorphisms

$$
f_{*}: H_{*}^{\kappa_{n}}\left(S_{n}\right) \longrightarrow H_{*}^{\kappa_{n}}\left(S_{n}\right) \text {. }
$$

Definition of degree of $f$ can be given similarly in algebraic topology (see [15]). We see that $f_{*}$ must be of the form

$$
f_{*}([x])=m[x]
$$

for some fixed $m \in \mathbb{Z}$, where $[x]$ is a generator of $H_{*}^{k_{n}}\left(S_{n}\right)$. This $m$ is the called the degree of $f$.

Theorem 3.11. Let $S_{n}$ be a digital $n$-sphere where $n \in\{1,2\}$. If $f: S_{n} \longrightarrow S_{n}$ is a map of degree $m \neq 1$, then $f$ has fixed point.

Proof. Let $f$ be a map of degree $m \neq 1$.

$$
f_{*}: H_{*}^{\kappa}\left(S_{n}\right) \longrightarrow H_{*}^{\mathcal{K}}\left(S_{n}\right)
$$

The trace of $f_{*}$ must be $m$. Since the trace of $f_{0}: H_{0}^{\kappa}\left(S_{n}\right) \longrightarrow H_{0}^{\kappa}\left(S_{n}\right)$ is 1 , we have

$$
\begin{aligned}
\lambda(f) & =\sum_{i=0}^{1}(-1)^{i} \operatorname{tr}\left(f_{i}\right) \\
& =\operatorname{tr}\left(f_{0}\right)-\operatorname{tr}\left(f_{1}\right) \\
& =1-m .
\end{aligned}
$$

Since $m \neq 1$, we get $\lambda(f) \neq 0$. By Theorem 3.4, $f$ has a fixed point theorem. 
Proposition 3.12. Let $(X, \kappa)$ be digital image and $f:(X, \kappa) \longrightarrow(X, \kappa)$ be a map. If

$$
\begin{gathered}
f_{*}: H_{*}^{\kappa}(X) \longrightarrow H_{*}^{\kappa}(X) \\
z \longmapsto f_{*}(z)=k z
\end{gathered}
$$

where $k$ is an integer, then $\lambda(f)=k \chi(X)$.

Proof. It is easy to see that

$$
\begin{aligned}
\lambda\left(1_{X}\right) & =\sum_{q=0}^{\infty}(-1)^{q} \operatorname{tr}\left(\left(1_{X}\right)_{*}\right) \\
& =\sum_{q=0}^{\infty}(-1)^{q} \operatorname{rank} H_{q}^{\kappa}(X) \\
& =\chi(X) .
\end{aligned}
$$

As a result, we get

$$
\begin{aligned}
k \chi(X) & =k \sum_{q=0}^{\infty}(-1)^{q} \operatorname{rank} H_{q}^{\kappa}(X) \\
& =\sum_{q=0}^{\infty}(-1)^{q} k \cdot \operatorname{rank} H_{q}^{\kappa}(X) \\
& =\sum_{q=0}^{\infty}(-1)^{q} \operatorname{tr}\left(f_{*}\right) \\
& =\lambda(f) .
\end{aligned}
$$

Definition 3.13. If $x=\left(x_{1}, \ldots, x_{n+1}\right) \in S_{n}$, its antipode is $-x=\left(-x_{1}, \ldots,-x_{n+1}\right)$. The antipodal map $\alpha=\alpha^{n}: S_{n} \longrightarrow S_{n}$ is defined by $\alpha(x)=-x$.

Theorem 3.14. Let $\alpha_{i}:\left(S_{i}, \kappa_{i}\right) \longrightarrow\left(S_{i}, \kappa_{i}\right)$ be the antipodal map between two digital $i$-spheres where $i=\{1,2\}$ and $\kappa_{i}=\{4,6\}$. Then $\alpha_{i}$ has degree $(-1)^{i+1}$.

Proof. By definition, we have $\operatorname{deg}\left(\alpha_{i}\right)=d$, where $\left(\alpha_{i}\right)_{*}: H_{i}^{\kappa_{i}}\left(S_{i}\right) \longrightarrow H_{i}^{\kappa_{i}}\left(S_{i}\right)$ is multiplication by $d$. Thus we get

$$
\operatorname{tr}\left(\alpha_{i}\right)_{*}=d=\operatorname{deg}\left(\alpha_{i}\right)
$$

so that

$$
\lambda\left(\alpha_{i}\right)=1+(-1)^{i} d
$$

but from Theorem 3.4, we have $\lambda\left(\alpha_{i}\right)=0$ because the antipodal map has no fixed points. As a result, $d=(-1)^{i+1}$. 
Theorem 3.15. Let $h:\left(S_{1}, 4\right) \longrightarrow\left(S_{1}, 4\right)$ be a digital $(4,4)$-continuous map. If $h$ is digitally nullhomotopic, then $h$ has a fixed point.

Proof. Since $h:\left(S_{1}, 4\right) \longrightarrow\left(S_{1}, 4\right)$ is digitally nullhomotopic, $h \simeq_{(4,4)} c$ where $c$ is a constant map on $\left(S_{1}, 4\right)$. By Theorem 3.5, we have $h_{*}=c_{*}$. Since the Lefschetz number of $c$ is

$$
\lambda(c)=\sum_{i=0}^{\infty}(-1)^{i} \operatorname{tr}\left(c_{*}\right)=1,
$$

we conclude that $h$ has a fixed point.

Corollary 3.16. If $h:\left(S_{1}, 4\right) \longrightarrow\left(S_{1}, 4\right)$ is defined by $h\left(c_{i}\right)=c_{i+1}$ where $c_{i} \in S_{1}$, then $h$ is not nullhomotopic.

Proof. It's clear that $h$ has not a fixed point. So from Theorem 3.15, we conclude that $h$ is not nullhomotopic.

Corollary 3.17. There is at least one digital $(4,4)$-continuous map

$$
\begin{aligned}
h:\left(S_{1}, 4\right) & \longrightarrow\left(S_{1}, 4\right) \\
c_{i} & \longmapsto h\left(c_{i}\right)=c_{i+4}(\bmod 8)
\end{aligned}
$$

for all $c_{i} \in S_{1}$ which maps some point $x \in S_{1}$ to its antipode $-x$.

Lemma 3.18. If $A \subset S_{1}$ is digital homeomorphic to $I^{k}$ for $k \in\{0,1\}$ where $I=[0,1]_{\mathbb{Z}}$, then $H_{q}^{4}\left(S_{1}-A\right)=0$ for all $q \geq 0$.

Proof. Since $S_{1}-A$ is digital 4-contractible image, digital homology groups of $S_{1}-A$ and one-point digital image are digital isomorphic. Thus we have

$$
H_{q}^{4}\left(S_{1}-A\right)=0
$$

for all $q \geq 0$.

Ege et al. in [12] give definition and properties of relative homology groups in digital images. We now would like to discuss the relative Lefschetz number for digital images.

Definition 3.19. Let $f:(X, A) \longrightarrow(X, A)$ be a digital map, where $(A, \kappa)$ is a digital subcomplex of $(X, \kappa)$. The map $f$ induces homomorphisms

$$
f_{*}: H_{q}^{\kappa}(X, A) \longrightarrow H_{q}^{\kappa}(X, A) .
$$

The relative Lefschetz number $\lambda(f ; X, A)$ is defined by

$$
\lambda(f ; X, A)=\sum_{q=0}^{n}(-1)^{q} \operatorname{tr}\left(f_{*}\right),
$$

where $f_{*}: H_{q}^{\kappa}(X, A) \longrightarrow H_{q}^{\kappa}(X, A)$. 
Example 3.20. We compute the relative Lefschetz number of $\left(M S S_{6}^{\prime}, A\right)$ where

$$
A=\left\{c_{0}, c_{1}, c_{2}, c_{3}\right\} \subset M S S_{6}^{\prime}
$$

has 6-adjacency. Ege et al. [12] show that for all $q \geq 0, H_{q}^{4}\left(M_{S S_{6}^{\prime}}^{\prime} A\right)=0$. Let $f:\left(M_{S S_{6}}^{\prime}, A\right) \longrightarrow\left(M_{S S_{6}}^{\prime}, A\right)$ be a digital map. The relative Lefschetz number of $f$ is given by

$$
\lambda\left(f ; \mathrm{MSS}_{6}^{\prime}, A\right)=\sum_{q=0}^{n}(-1)^{q} \operatorname{tr}\left(f_{*}\right)=0,
$$

where $f_{*}: H_{q}^{6}\left(M_{S} S_{6}^{\prime}, A\right) \longrightarrow H_{q}^{6}\left(M S S_{6}^{\prime}, A\right)$.

We now would like to deal with a property on the relative Lefschetz number, which is satisfied in algebraic topology but is not satisfied in digital images. The property is given as follows. Let $(X, \kappa)$ be a digital complex, $A$ and $B$ be digital subcomplexes of $X$. Then

$$
\lambda(f)=\lambda\left(f_{A}\right)+\lambda\left(f_{B}\right)-\lambda\left(f_{A \cap B}\right),
$$

where $f: X \longrightarrow X, f_{A}: A \longrightarrow A, f_{B}: B \longrightarrow B$ and $f_{A \cap B}: A \cap B \longrightarrow A \cap B$. The above property is not satisfied in digital images. Let's show it by an example.

Example 3.21. Let $X=M_{S S_{6}^{\prime}}^{\prime} A=\left\{c_{0}, c_{1}, c_{2}, c_{3}\right\}, B=\left\{c_{4}, c_{5}, c_{6}, c_{7}\right\}$ and $A \cap B=\varnothing$. Moreover, we take maps

$$
f: X \longrightarrow X, f_{A}: A \longrightarrow A, f_{B}: B \longrightarrow B \text { and } f_{A \cap B}: A \cap B \longrightarrow A \cap B .
$$

Since $A$ and $B$ are digital simple closed 4-curves [1],

$$
H_{q}^{4}(A)=H_{q}^{4}(B)= \begin{cases}\mathbb{Z}, & q=0,1 \\ 0, & q \neq 0,1\end{cases}
$$

Therefore, we have the following results.

$$
\begin{aligned}
\lambda(f) & =1-5=-4, \\
\lambda\left(f_{A}\right) & =1-1=0, \\
\lambda\left(f_{B}\right) & =1-1=0, \\
\lambda\left(f_{A \cap B}\right) & =0 .
\end{aligned}
$$

From the following inequality

$$
-4 \neq 0+0-0
$$

we conclude that the equality

$$
\lambda(f)=\lambda\left(f_{A}\right)+\lambda\left(f_{B}\right)-\lambda\left(f_{A \cap B}\right)
$$

does not yield for digital images.

Theorem 3.5 can be given for relative digital images as follows. 
Theorem 3.22. Let $(X, \kappa)$ be a digital complex and $A \subset X$ be a digital subcomplex. If $f:(X, A, \kappa) \longrightarrow(X, A, \kappa)$ has $\lambda(f ; X, A) \neq 0$, then any map homotopic to $f$ has a fixed point.

Proof. Let $g:(X, A, \kappa) \longrightarrow(X, A, \kappa)$ be $(\kappa, \kappa)$-homotopic to $f$. From homotopy axiom, we have

$$
f_{*}=g_{*}: H_{*}^{\kappa}(X, A) \longrightarrow H_{*}^{\kappa}(X, A) .
$$

We conclude that

$$
\lambda(f ; X, A)=\sum_{q=0}^{n}(-1)^{q} \operatorname{tr}\left(f_{*}\right)=\sum_{q=0}^{n}(-1)^{q} \operatorname{tr}\left(g_{*}\right)=\lambda(g ; X, A) .
$$

As a result, $g$ has a fixed point.

Definition 3.23. Let $(X, \kappa)$ be a digital image and $f:(X, \kappa) \longrightarrow(X, \kappa)$ be a digital map. The reduced Lefschetz number $\tilde{\lambda}(f)$ is defined by

$$
\tilde{\lambda}(f)=\lambda(f)-1,
$$

where $\lambda(f)$ is the Lefschetz number of $f$.

Example 3.24. Let's compute the reduced Lefschetz number of

$$
f: M S S_{18}^{\prime} \longrightarrow M S S_{18}^{\prime}
$$

where $M S S_{18}^{\prime}$ and its digital homology groups are given in Example 2.11. From Theorem 2.17, we know that

$$
\begin{gathered}
H_{0}^{18}\left(M S S_{18}^{\prime}\right) \cong \widetilde{H_{0}^{18}}\left(M S S_{18}^{\prime}\right) \oplus \mathbb{Z}, \\
H_{q}^{18}\left(M S S_{18}^{\prime}\right)=\widetilde{H_{q}^{18}}\left(M S S_{18}^{\prime}\right), \quad q \geq 1 .
\end{gathered}
$$

Since $H_{0}^{18}\left(M_{S S_{18}^{\prime}}^{\prime}\right) \cong \mathbb{Z}$, we have $\widetilde{H_{0}^{18}}\left(M_{S S}^{\prime}{ }_{18}\right)=0$. So the reduced homology groups of $M S S_{18}^{\prime}$ are

$$
\widetilde{H_{q}^{18}}\left(M_{S S S_{18}^{\prime}}^{\prime}\right)= \begin{cases}\mathbb{Z}, & q=2 \\ 0, & q \neq 2\end{cases}
$$

The Lefschetz number of $f$ is

$$
\lambda(f)=\sum_{q=0}^{\infty}(-1)^{q} \operatorname{tr}\left(f_{q}\right)=1-0+1-0+\ldots=2,
$$

where $f_{q}: H_{q}^{18}\left(M_{S S_{18}^{\prime}}^{\prime}\right) \longrightarrow H_{q}^{18}\left(M_{S S_{18}^{\prime}}^{\prime}\right)$. As a result, the reduced Lefschetz number of $f$ is

$$
\tilde{\lambda}(f)=\lambda(f)-1=2-1=1 .
$$




\section{Conclusion}

The essential aim of this study is to determine some important applications of fixed point theory for a digital image. We study the relation between the Euler characteristic and the Lefschetz number. We deal with some characterizations about the Lefschetz number. We give also calculations about theorems and properties. Relative and reduced Lefschetz number is defined for digital images. We expect that properties in the paper will be useful for fixed point theory.

\section{References}

[1] H. Arslan, I. Karaca and A. Oztel, "Homology groups of n-dimensional digital images", XXI. Turkish National Mathematics Symposium, B1-13, 2008.

[2] L. Boxer, "Digitally continuous functions", Pattern Recognition Letters 15, 833-839, 1994.

[3] L. Boxer, "A classical construction for the digital fundamental group", Journal of Mathematical Imaging and Vision 10, 51-62, 1999.

[4] L. Boxer, "Properties of digital homotopy", Journal of Mathematical Imaging and Vision 22, 19-26, 2005.

[5] L. Boxer, "Homotopy properties of sphere-like digital images", Journal of Mathematical Imaging and Vision 24, 167-175, 2006.

[6] L. Boxer, Digital products, wedges and covering spaces, Journal of Mathematical Imaging and Vision 25, 169-171, 2006.

[7] L. Boxer, I. Karaca and A. Oztel, "Topological invariants in digital images", Journal of Mathematical Sciences: Advances and Applications 11(2), 109-140, 2011.

[8] E. Demir and I. Karaca, Simplicial Homology Groups of Certain Digital Surfaces, Hacettepe Journal of Mathematics and Statistics, In press, 2014.

[9] A. Granas and J. Dugundji, "Fixed Point Theory", Springer, 2003.

[10] O. Ege and I. Karaca, "The Lefschetz Fixed Point Theorem for Digital Images", Fixed Point Theory and Applications, doi:10.1186/10.1186/16871812-2013-253, 2013.

[11] O. Ege and I. Karaca, "Fundamental properties of simplicial homology groups for digital images", American Journal of Computer Technology and Application 1 No.2, 25-42, 2013.

[12] O. Ege, I. Karaca and M.E. Ege, "Relative Homology Groups of Digital Images", Applied Mathematics and Information Science, 8(5), 2337-2345, 2014. 
[13] G.T. Herman, "Oriented surfaces in digital spaces", CVGIP: Graphical Models and Image Processing 55, 381-396, 1993.

[14] I. Karaca and O. Ege, Some results on simplicial homology groups of 2D digital images, International Journal of Information and Computer Science 1 no.8, 198-203, 2012.

[15] E. Spanier, Algebraic Topology, McGraw-Hill, New York, 1966.

Department of Mathematics, Celal Bayar University, Muradiye, Manisa, 45140, Turkey email:ozgur.ege@cbu.edu.tr url: http://www.bayar.edu.tr/ fef/matematik/ozgecmis/oege.pdf Tel.:+90 2362013228

Departments of Mathematics, Ege University, Bornova, Izmir, 35100, Turkey email:ismet.karaca@ege.edu.tr url:fen.ege.edu.tr $\backslash \sim$ ismetkaraca 